\title{
Derivation of generalized Einstein's equations of gravitation in some non-inertial reference frames based on the theory of vacuum mechanics
}

\author{
Xiao-Song Wang \\ Institute of Mechanical and Power Engineering, Henan Polytechnic University, Jiaozuo, Henan Province, 454000, China \\ (Dated: Dec. 15, 2020)
}

\begin{abstract}
When solving the Einstein's equations for an isolated system of masses, V. Fock introduces harmonic reference frame and obtains an unambiguous solution. Further, he concludes that there exists a harmonic reference frame which is determined uniquely apart from a Lorentz transformation if suitable supplementary conditions are imposed. It is known that wave equations keep the same form under Lorentz transformations. Thus, we speculate that Fock's special harmonic reference frames may have provided us a clue to derive the Einstein's equations in some special class of non-inertial reference frames. Following this clue, generalized Einstein's equations in some special non-inertial reference frames are derived based on the theory of vacuum mechanics. If the field is weak and the reference frame is quasi-inertial, these generalized Einstein's equations reduce to Einstein's equations. Thus, this theory may also explain all the experiments which support the theory of general relativity. There exist some differences between this theory and the theory of general relativity.
\end{abstract}

Keywords: Einstein's equations; gravitation; general relativity; principle of equivalence; gravitational aether; vacuum mechanics.

\section{INTRODUCTION}

The Einstein's field equations of gravitation are valid in all reference frames is a fundamental assumption in the theory of general relativity [1-3]. R. P. Feynman once said:" What I cannot create, I do not understand." ([4], p. xxxii). New theories which can derive Einstein's field equations may be interesting. The reasons may be summarized as follows.

1. Many attempts to reconcile the theory of general relativity and quantum mechanics by using the techniques in quantum electrodynamics meet some mathematical difficulties ([5], p. 101). J. Maddox speculates that the failure of the familiar quantization procedures to cope with Einstein's equations may stem from two possible reasons. One possibility is that Einstein's equations are incomplete. The other possible reason may be that some underlying assumptions in Einstein's theory about the character of the space or time may be not suitable ([5], p. 101).

2. The value of the cosmological constant is a puzzle [6]. In 1917, A. Einstein thought that his equations should be revised to be ([3], p. 410)

$$
R_{\mu \nu}-\frac{1}{2} g_{\mu \nu} R+\Lambda g_{\mu \nu}=-\kappa T_{\mu \nu}^{m}
$$

where $g_{\mu \nu}$ is the metric tensor of a Riemannian spacetime, $R_{\mu \nu}$ is the Ricci tensor, $R \equiv g^{\mu \nu} R_{\mu \nu}$ is the scalar curvature, $g^{\mu \nu}$ is the contravariant metric tensor, $\kappa$ is a constant, $T_{\mu \nu}^{m}$ is the energy-momentum tensor of a matter system, $\Lambda$ is the cosmological constant.

However, it seems that the cosmological constant $\Lambda$ is unnecessary when Hubble discovered the expansion of the universe. Thus, Einstein abandoned the term $\Lambda g_{\mu \nu}$ in Eqs. (1) and returned to his original equations ([3], p. 410). The value of the cosmological constant $\Lambda$ is also related to the energy-momentum tensor of vacuum ([3], p. 411). Theoretical interpretation of the small value of $\Lambda$ is still open [6].

3. The problem of the existence of black hole is interesting [7]. Einstein believed that black hole cannot exist in the real world [8]. Recently, the Event Horizon Telescope Collaboration (EHTC) reconstructed eventhorizon-scale images of the supermassive black hole candidate in the center of the giant elliptical galaxy M87 [9]. EHTC reports that the observed image is consistent with predictions for the shadow of a Kerr black hole based on the theory of general relativity.

4. The existences and characters of dark matter and dark energy are still controversy, refers to, for instance, [10-14].

5. The existence and characters of gravitational aether are still not clear. Sir I. Newton pointed out that his inverse-square law of gravitation did not touch on the mechanism of gravitation ([15], p. 28;[16], p. 91). He conjectured that gravitation may be explained based on the action of an aether pervading the space ([15], p. 28; [16], p. 92 ). In the years 1905-1916, Einstein abandoned the concepts of electromagnetic aether and gravitational aether in his theory of relativity ([17], p. 27-61). However, H. A. Lorentz believed that general relativity could be reconciled with the concept of an ether at rest and wrote a letter to A. Einstein ([17], p. 65). Einstein changed his view later and introduced his new concept of ether ([17], p. 63-113). However, Einstein did not tell us how to derive his equations theoretically based on his new concept of the gravitational aether.

6. Whether Newton's gravitational constant $G_{N}$ depends on time and space is still not clear. It is known that $G_{N}$ is a constant in Newton's and Einstein's theory of gravitation. P. A. M. Dirac speculates that $G_{N}$ may depend on time based on his large number hypothesis [18]. R. P. Feynman thought that if $G_{N}$ decreases on time, then the earth's temperature a billion years ago was about $48^{\circ} \mathrm{C}$ higher than the present temperature 
([4], p. 9).

Furthermore, there exist some other problems related to the theories of gravity, for instance, gravitational waves [19], the speed of light in vacuum [20], the definition of inertial system, origin of inertial force, the velocity of the propagation of gravity [21], the velocity of individual photons [22], unified field theory, etc.

The gravitational interaction seems to differ in character from other interactions. The existing theories of gravity still face the aforementioned difficulties. Thus, it seems that new ideas about the gravitational phenomena are needed. Following Einstein [23], it may be better for us to keep an open and critical mind to explore all possible theories about gravity.

In 2012, M. J. Dupre and F. J. Tipler propose an aether theory of general relativity [24]. Recently, generalized Einstein's equations of gravitational fields in inertial reference frames are derived based on a sink flow model of particles [25]. However, the cases of non-inertial reference frames are not discussed in Ref. [25]. The purpose of this manuscript is to propose a derivation of the Einstein's equations in some non-inertial reference frames based on the theory of vacuum mechanics [25-28].

\section{A BRIEF INTRODUCTION OF THE THEORY OF VACUUM MECHANICS}

Ancient people believe that there is a kind of continuously distributed substance which fills every corner of the space. Ancient Egyptians called this substance as nun ([29], p. 172). According to ancient Egyptians, the nun is a primeval watery darkness which is continuously distributed surround the world ([29], p. 172). Ancient Greeks, such as Thales and Anaximenes, believe that everything in the universe is made of a kind of fundamental substance named aether.

The thought that our universe is an infinite hierarchy which has universes within universes without end is a charming idea. Astonished by this attractive idea and inspired by the aforementioned ideas, we propose the following mechanical model of the universe.

Matter is composed of molecules. Molecules are constructed by atoms. Atoms are formed by elementary particles. Modern experiments, for instance, the Casimir effect, have shown that vacuum is not empty. Therefore, new considerations on the old concept of aether may be needed.

Thomson's analogies between electrical phenomena and elasticity helped J. C. Maxwell to establish a mechanical model of electrical phenomena [15]. Following J. C. Maxwell, we introduce the following assumption [26].

Assumption 1 We suppose that vacuum is filled with a kind of continuously distributed matter which may be called the $\Omega(1)$ substratum, or the electromagnetic aether.

The idea that all microscopic particles are sink flows in a fluidic substratum has been proposed by many researchers in the history, for instance, J. C. Maxwell ([15], p. 243), B. Riemann ([30], p. 507), H. Poincaré ([31], p. 171), J. C. Taylor ([32], p. 431-436). Thus, we suppose that electric charges are sources or sinks of the $\Omega(1)$ substratum [26]. Maxwell's equations in vacuum are derived by methods of continuum mechanics based on a mechanical model of vacuum and a source or sink flow model of electric charges [26]. The electromagnetic aether behaves as a visco-elastic continuum [26]. Maxwell's equations approximate the macroscopic behavior of the $\Omega(1)$ particles, in analogy to the way that classical elastic mechanics approximates the macroscopic behavior of the atoms of solid materials.

Descartes interpreted the celestial motions of celestial bodies based on the hypothesis that the universe is filled by a fluidic vortex aether [15]. Since Newton's law of gravitation was published in 1687 [33], this action-at-adistance theory was criticized by the French Cartesian [34]. Sir I. Newton tried to obtain a derivation of his law based on Descartes' scientific research program. At last, he proved that Descartes' vortex aether hypothesis could not explain celestial motions properly [33]. Newton himself even suggested an explanation of gravity based on the action of an aetherial medium pervading the space ([15], p. 28). Euler attempted to explain gravity based on some hypotheses of a fluidic aether [34]. Following Descartes, we introduce the following assumption [27].

Assumption 2 We suppose that vacuum is filled by an extremely thin medium which may be called the $\Omega(0)$ substratum, or the gravitational aether.

Thus, two sinks in the $\Omega(0)$ substratum are found to attract with each other according to the inverse-square law of gravitation [27]. A feature of this theory is that the gravitational constant depends on time and the location in space.

The particles that constitute the $\Omega(1)$ substratum may be called the $\Omega(1)$ particles. Lord Kelvin believes that the electromagnetic aether must also generate gravity [24, 35]. Following Lord Kelvin, we introduce the following assumption.

Assumption 3 We suppose that there exist a kind of basic sinks of the $\Omega(0)$ substratum, which may be called monads after Leibniz. The $\Omega(1)$ particles and elementary particles are formed of monads.

Since monads, the $\Omega(1)$ particles, elementary particles are sinks of the $\Omega(0)$ substratum, they attract with each other according to Newton's law of gravitation [27].

There exists an universal drag force exerted on each sink of the $\Omega(0)$ substratum [27]. Therefore, each monad, each $\Omega(1)$ particle and each elementary particle, as sinks of the $\Omega(0)$ substratum, will experience the universal drag force. On the other hand, all the monads, $\Omega(1)$ particles and elementary particles are undertaking stochastic movements. Based on this universal damping force and some assumptions, microscopic particles are 
found to obey a generalized non-relativistic Schrödinger equation [28].

For convenience, we may call these theories [25-28] as the theory of vacuum mechanics. Vacuum mechanics is a physical theory which attempts to derive some basic physical laws based on a new mechanical model of vacuum and particles.

\section{EQUIVALENCE BETWEEN THE INERTIAL MASS AND THE GRAVITATIONAL MASS}

Proposition 1 The inertial mass of a microscopic particle equals its gravitational mass.

Proof of Proposition 1. Newton's law of gravitation can be written as ([36], p. 2)

$$
\mathbf{F}_{12}=-G_{N} \frac{m_{g 1} m_{g 2}}{r^{2}} \hat{\mathbf{r}}_{12}
$$

where $\mathbf{F}_{12}(t)$ denotes the force exerted on the particle with gravitational mass $m_{g 2}$ by the particle with gravitational mass $m_{g 1}, m_{g 1}$ and $m_{g 2}$ are the gravitational masses of two particles, $G_{N}$ is Newton's gravitational constant, $\hat{\mathbf{r}}_{12}$ denotes the unit vector directed along the line from the particle with mass $m_{g 1}$ to the particle with mass $m_{g 2}, r$ is the distance between the two particles.

In 2008, we show that the force $\mathbf{F}_{12}(t)$ exerted on the particle with inertial mass $m_{i 2}(t)$ by the velocity field of the $\Omega(0)$ substratum induced by the particle with inertial mass $m_{i 1}(t)$ is [27]

$$
\mathbf{F}_{12}(t)=-\gamma_{N}(t) \frac{m_{i 1}(t) m_{i 2}(t)}{r^{2}} \hat{\mathbf{r}}_{12}
$$

where

$$
\gamma_{N}(t)=\frac{\rho_{0} q_{0}^{2}}{4 \pi m_{0}^{2}(t)},
$$

$\rho_{0}$ is the density of the $\Omega(0)$ substratum, $m_{0}(t)$ is the inertial mass of a monad at time $t,-q_{0}\left(q_{0}>0\right)$ is the strength of the monad.

Suppose that $G_{N}=\gamma_{N}(t)$. Comparing Eq. (2) and Eq. (3), we have

$$
m_{i 1} m_{i 2}=m_{g 1} m_{g 2}
$$

Now we study a gravitational system of two protons. According to Eq. (5), we have

$$
m_{i p}^{2}=m_{g p}^{2}
$$

where $m_{i p}$ and $m_{g p}$ are the inertial mass and gravitational mass of a proton respectively.

Noticing $m_{i p}>0$ and $m_{g p}>0$, Eq. (6) can be written as

$$
m_{i p}=m_{g p}
$$

Eq. (7) shows that the inertial mass $m_{i p}$ of a proton equals its gravitational mass $m_{g p}$. Similarly, we can demonstrate that the inertial mass of another type of microscopic particle equals its gravitational mass.

This result is called the principle of equivalence in the theory of general relativity [1-3].

\section{THE DYNAMICAL GRAVITATIONAL POTENTIALS IN INERTIAL REFERENCE FRAMES}

The purpose of this section is to review the mathematical forms of the dynamical gravitational potentials in inertial reference frames. These results may provide us some clues to explore possible mathematical model$\mathrm{s}$ of inertial potential and inertial force Lagrangian in non-inertial reference frames, which are introduced in the next section.

We introduce a Cartesian coordinate system $\{0, x, y, z\}$ for a three-dimensional Euclidean space that attached to the $\Omega(1)$ substratum. Let $\{0, t\}$ be a one-dimensional time coordinate. For convenience, we introduce the following Galilean coordinate system $x^{0} \equiv$ ct, $\quad x^{1} \equiv$ $x, \quad x^{2} \equiv y, \quad x^{3} \equiv z$. Let $\eta_{\mu \nu}$ denotes the metric tensor of the Minkowski spacetime.

We will use Greek indices $\alpha, \beta, \mu, \nu$, etc., to denote the range $\{0,1,2,3\}$ and Latin indices $i, j, k$, etc., to denote the range $\{1,2,3\}$. Einstein's summation convention will be used, i.e., any repeated Greek superscript or subscript appearing in a term of an equation is to be summed from 0 to 3 .

The definition of the strength $\mathbf{g}$ of a gravitational field is $([37]$, p. 24)

$$
\mathbf{g}=\frac{\mathbf{F}_{g}}{m_{\text {test }}}
$$

where $m_{\text {test }}$ is the mass of a test point particle, $\mathbf{F}_{g}$ is the gravitational force exerted on the test point particle by a gravitational field.

According to Newton's second law, we have

$$
\mathbf{F}_{g}=m_{\text {test }} \mathbf{a},
$$

where $\mathbf{a}$ is the acceleration of the test point particle.

Comparing Eq. (9) and Eq. (8), we have

$$
\mathrm{g}=\mathbf{a} \text {. }
$$

The definition of the acceleration $\mathbf{a}$ is ([37], p. 24)

$$
a_{i}=\gamma_{i k} \frac{d^{2} x^{k}}{d t^{2}}
$$

where

$$
\gamma_{i k}=-g_{i k}+\frac{g_{0 i} g_{0 k}}{g_{00}}
$$

$g_{\mu \nu}$ is the metric tensor of a Riemannian spacetime. 
Based on the time tracks of free particles described by geodesic curves in Minkowski spacetime, we have the following results ([1], p. 279;[37], p. 26)

$$
a_{i}=-\frac{\partial \Pi}{\partial x^{i}}-c \sqrt{1+\frac{2 \Pi}{c^{2}}} \frac{\partial \gamma_{i}}{\partial t}
$$

where

$$
\Pi=-\frac{1-g_{00}}{2} c^{2}, \quad \gamma_{i}=-\frac{g_{i 0}}{\sqrt{g_{00}}} .
$$

If we suppose that $\partial g_{00} / \partial t \approx 0$, then Eqs. (13) can also be written as

$$
a_{i}=c^{2} \frac{\partial}{\partial x^{i}}\left(\frac{1-g_{00}}{2}\right)-c^{2} \frac{\partial\left(-g_{i 0}\right)}{\partial(c t)} .
$$

\section{INERTIAL POTENTIAL AND INERTIAL FORCE LAGRANGIAN IN NON-INERTIAL REFERENCE FRAMES}

According to the theory of general relativity [2, 3], the Einstein's equations are valid not only in inertial reference frames but also in non-inertial reference frames. Thus, it is needed to explore the possibility to derive the Einstein's equations in non-inertial reference frames.

When solving the Einstein's equations for an isolated system of masses, V. Fock introduces harmonic reference frame and obtains an unambiguous solution ([38], p. 369). Furthermore, in the case of an isolated system of masses, he concludes that there exists a harmonic reference frame which is determined uniquely apart from a Lorentz transformation if suitable supplementary conditions are imposed ([38], p. 373). It is known that wave equations keep the same form under Lorentz transformations [1]. From Eqs. (93) in Ref. [25], we notice that the field equations of gravitation in inertial reference frames in a Minkowski spacetime are wave equations. Thus, we speculate that Fock's special harmonic reference frames may have provided us a clue to derive the Einstein's equations in some special class of non-inertial reference frames.

We introduce an arbitrary non-inertial coordinate system $\left(x^{\prime 0}, x^{\prime 1}, x^{\prime 2}, x^{\prime 3}\right)$ and denote it as $S_{n}$. It is known that a particle in a non-inertial reference frame will experience an inertial force. Unfortunately, we have no knowledge about the origin of inertial forces.

For convenience, we introduce the following definition of matter system.

Definition 1 A matter system is a system of a number of elementary particles, or continuously distributed elementary particles.

The equivalence between inertial mass and gravitational mass implies that to some degree gravitational forces behave in the same way as inertial forces ([36], p. 17). Thus, we speculate that inertial forces may originate from the interactions between matter systems and vacuum. Therefore, we introduce the following assumption.
Assumption 4 The inertial force exerted on a matter system in a non-inertial reference frame stems from the interactions between the matter system and vacuum.

Based on Assumption 4, we introduce the following concepts for inertial forces, which are similar to those concepts for gravitational interactions.

Definition 2 Inertial potential $\psi_{\mu \nu}^{\text {iner }}$ is an interaction potential between a matter system and vacuum resulting from the inertial force $\mathbf{F}_{\text {iner }}$ exerted on the matter system by vacuum in a non-inertial reference frame $S_{n}$.

Definition 3 Inertial force Lagrangian $L_{\text {iner }}$ is an interaction Lagrangian between a matter system and vacuum resulting from the inertial force $\mathbf{F}_{\text {iner }}$ exerted on the matter system by vacuum in a non-inertial reference frame $S_{n}$.

Now our task is to explore possible models of inertial potential $\psi_{\mu \nu}^{\text {iner }}$ and inertial force Lagrangian $L_{\text {iner }}$. Let $\eta_{\mu \nu}^{\prime}$ denotes the metric tensor of the non-inertial reference frame $S_{n}$. Suppose that $\partial \eta_{00}^{\prime} / \partial x^{\prime 0} \approx 0$. Then, following similar methods as in the derivation of Eqs. (15), we obtain the following relationship for the inertial acceleration $\mathbf{a}$ of a test point particle in the non-inertial reference frame $S_{n}$

$$
a_{i}=c^{2} \frac{\partial}{\partial x^{\prime i}}\left(\frac{1-\eta_{00}^{\prime}}{2}\right)-c^{2} \frac{\partial\left(-\eta_{i 0}^{\prime}\right)}{\partial x^{\prime 0}}
$$

If $\eta_{i 0}^{\prime}$ are time-independent, the inertial acceleration a of the test point particle in Eqs. (16) simplifies to ([1], p. 280)

$$
a_{i}=c^{2} \frac{\partial}{\partial x^{\prime i}}\left(\frac{1-\eta_{00}^{\prime}}{2}\right) .
$$

Using Eqs. (17), the inertial force $\mathbf{F}_{\text {iner }}$ exerted on the test point particle can be written as

$$
\mathbf{F}_{\text {iner }}=m \mathbf{a}=m c^{2} \nabla\left(\frac{1-\eta_{00}^{\prime}}{2}\right),
$$

where $m$ is the mass of the test point particle, $\nabla=$ $\mathbf{i} \partial / \partial x^{1}+\mathbf{j} \partial / \partial x^{\prime 2}+\mathbf{k} \partial / \partial x^{\prime 3}$ is the Hamilton operator, $\mathbf{i}, \mathbf{j}, \mathbf{k}$ are three unit vectors directed along the coordinate axes.

From Eq. (18), the inertial force Lagrangian of a system of vacuum and the test point particle can be written as

$$
L_{\text {iner1 }}=-m c^{2}\left(\frac{1-\eta_{00}^{\prime}}{2}\right) .
$$

Therefore, the inertial force Lagrangian of a system of vacuum and continuously distributed particles can be written as

$$
L_{\text {iner }}=-\rho_{m} c^{2}\left(\frac{1-\eta_{00}^{\prime}}{2}\right)
$$


where $\rho_{m}$ is the rest mass density of the continuously distributed particles.

Let $T_{m}^{\prime} \mu \nu$ denotes the contravariant energy-momentum tensor of the continuously distributed particles system. If the inertial force is small enough, then we may regard this non-inertial reference frame $S_{n}$ as an inertial reference frame approximately. Thus, we have $T_{m}^{\prime 00} \approx \rho_{m} c^{2}[25]$. Noticing $\eta_{00}=1$, the inertial force Lagrangian $L_{\text {iner }}$ in Eq. (20) can be written as

$$
L_{\mathrm{iner}} \approx f_{0} \psi_{00}^{\text {iner }} T_{m}^{\prime} 00
$$

where

$$
\psi_{00}^{\text {iner }}=-\frac{1}{2 f_{0}}\left(\eta_{00}-\eta_{00}^{\prime}\right) .
$$

Following Ref. [25], the parameter $f_{0}$ is

$$
f_{0}=\sqrt{\frac{2 \rho_{0} q_{0}^{2}}{m_{0}^{2} c^{4}}}=\sqrt{\frac{8 \pi \gamma_{N}}{c^{4}}} .
$$

Inspired by Eq. (21) and Eq. (22), we introduce the following assumption.

Assumption 5 Suppose that the inertial force Lagrangian $L_{\text {iner }}$ of a system of a free point particle and vacuum in the non-inertial reference frame $S_{n}$ can be written as

$$
L_{\text {iner }}=f_{0} \psi_{\mu \nu}^{\text {iner }} m u^{\prime \mu} u^{\prime \nu},
$$

where $m$ is the rest mass of the point particle, $u^{\prime \mu} \equiv$ $d x^{\prime} \mu / d \tau_{\eta^{\prime}}, \tau_{\eta^{\prime}}$ is the proper time,

$$
\psi_{\mu \nu}^{\text {iner }}=-\frac{1}{2 f_{0}}\left(\eta_{\mu \nu}-\eta_{\mu \nu}^{\prime}\right) .
$$

Following similar methods as in [25], we obtain the following result.

Proposition 2 Suppose that Assumption 5 is valid. Then, the equations of motion of a free point particle can be written as

$$
\frac{d^{2} x^{\prime \mu}}{d \tau_{\eta^{\prime}}^{2}}+C_{\nu \sigma}^{\prime} \frac{d x^{\prime} \nu}{d \tau_{\eta^{\prime}}} \frac{d x^{\prime} \sigma}{d \tau_{\eta^{\prime}}}=0
$$

where

$$
C_{\alpha \beta}^{\prime \prime} \equiv \frac{1}{2} \eta^{\prime \mu \nu}\left(\frac{\partial \eta_{\mu \alpha}^{\prime}}{\partial x^{\prime \beta}}+\frac{\partial \eta_{\mu \beta}^{\prime}}{\partial x^{\prime \alpha}}-\frac{\partial \eta_{\alpha \beta}^{\prime}}{\partial x^{\prime \mu}}\right)
$$

are the corresponding Christoffel symbols in the noninertial reference frame $S_{n}$.

Proof of Proposition 2. The Lagrangian of a free point particle in $S_{n}$ can be written as ([4], p. 57;[39])

$$
L_{0}^{\prime}=\frac{1}{2} m \frac{d x^{\prime} \mu}{d \tau_{\eta^{\prime}}} \frac{d x_{\mu}^{\prime}}{d \tau_{\eta^{\prime}}}=\frac{1}{2} m u^{\prime \mu} u_{\mu}^{\prime}=\frac{1}{2} m \eta_{\mu \nu}^{\prime} u^{\prime \mu} u^{\prime \nu}
$$

where $m$ is the rest mass of the point particle, $d \tau_{\eta^{\prime}} \equiv$ $\frac{1}{c} \sqrt{d x^{\prime} \mu d x_{\mu}^{\prime}}$ is the infinitesimal proper time interval, $u^{\prime \mu} \equiv d x^{\prime \mu} / d \tau_{\eta^{\prime}}$.

Ignoring the contravariant energy-momentum tensor of the $\Omega(1)$ substratum, i.e., $T_{\Omega(1)}^{\prime \mu \nu} \approx 0$ and using Eq. (28) and Eq. (24), the total Lagrangian $L_{\mathrm{p}}$ of a system of the $\Omega(0)$ substratum, the $\Omega(1)$ substratum and the point particle can be written as

$$
L_{\mathrm{p}}=L_{0}^{\prime}+L_{\text {iner }}=\frac{1}{2} m u^{\prime \mu} u_{\mu}^{\prime}+f_{0} \psi_{\mu \nu}^{\text {iner }} m u^{\prime \mu} u^{\prime \nu} .
$$

The Euler-Lagrange equations for the total Lagrangian $L_{\mathrm{p}}$ can be written as ([36], p. 111)

$$
\frac{\partial L_{\mathrm{p}}}{\partial x^{\prime \mu}}-\frac{d}{d \tau_{\eta^{\prime}}} \frac{\partial L_{\mathrm{p}}}{\partial u^{\prime \mu}}=0 .
$$

Putting Eq. (29) into Eqs. (30), we have

$\frac{d}{d \tau_{\eta^{\prime}}}\left[\left(\eta_{\mu \nu}+2 f_{0} \psi_{\mu \nu}^{\text {iner }}\right) \frac{d x^{\prime} \nu}{d \tau_{\eta^{\prime}}}\right]-f_{0} \frac{\partial \psi_{\alpha \beta}^{\text {iner }}}{\partial x^{\prime} \mu} \frac{d x^{\prime} \alpha}{d \tau_{\eta^{\prime}}} \frac{d x^{\prime} \beta}{d \tau_{\eta^{\prime}}}=0$.

Using Eq. (25), Eqs. (31) can be written as

$$
\frac{d}{d \tau_{\eta^{\prime}}}\left(\eta_{\mu \nu}^{\prime} \frac{d x^{\prime} \nu}{d \tau_{\eta^{\prime}}}\right)-\frac{1}{2} \frac{\partial \eta_{\alpha \beta}^{\prime}}{\partial x^{\prime} \mu} \frac{d x^{\prime} \alpha}{d \tau_{\eta^{\prime}}} \frac{d x^{\prime} \beta}{d \tau_{\eta^{\prime}}}=0 .
$$

Eqs. (32) represent a geodesic line in a Minkowski spacetime with a metric tensor $\eta_{\mu \nu}^{\prime}$, which can also be written as Eqs. (26) ([37], p. 50).

Eqs. (26) is a geodesic curve in a Minkowski spacetime. It is known that a geodesic curve is a straight line in a Minkowski spacetime ([40], p. 235). For instance, according to Newton's first law, a free particle moves along a straight line in the Galilean coordinates. Therefore, Assumption 5 may be supported by some experiments. Inspired by the inertial force Lagrangian for a free point particle in Eq. (24), we introduce the following assumption for a matter system and vacuum based on Assumption 3 .

Assumption 6 The inertial force Lagrangian $L_{\text {iner }}$ of a matter system and vacuum in the non-inertial reference frame $S_{n}$ can be written as

$$
L_{\text {iner }}=f_{0} \psi_{\mu \nu}^{\text {iner }}\left(T_{m}^{\prime} \mu \nu+T_{\Omega(1)}^{\prime \mu \nu}\right)+O\left[\left(f_{0} \psi_{\mu \nu}^{\text {iner }}\right)^{2}\right]
$$

where $T_{m}^{\prime \mu \nu}$ and $T_{\Omega(1)}^{\prime \mu \nu}$ are the contravariant energymomentum tensors of the matter and the $\Omega(1)$ substratum respectively, $O\left[\left(f_{0} \psi_{\mu \nu}^{\text {iner }}\right)^{2}\right]$ denotes those terms which are small quantities of the order of $\left(f_{0} \psi_{\mu \nu}^{\text {iner }}\right)^{2}$.

\section{FIELD EQUATIONS IN A SPECIAL CLASS OF NON-INERTIAL REFERENCE FRAMES}

Suppose that the transformation equations between a non-inertial coordinate system $\left(x^{\prime 0}, x^{1}, x^{\prime 2}, x^{\prime 3}\right)$ and 
the Galilean coordinates $(c t, x, y, z)$ are

$$
x^{\prime \alpha}=f^{\alpha}\left(x^{0}, x^{1}, x^{2}, x^{3}\right) .
$$

Following V. Fock ([38], p. 370-373), we introduce the following definition of a special class of reference frames.

Definition 4 Suppose that a coordinate system $\left(x^{\prime 0}, x^{\prime 1}, x^{\prime 2}, x^{\prime 3}\right)$ satisfies the following conditions: (1) every coordinates $x^{\prime \alpha}$ satisfies the d'Alembert's equation ([38], p. 369), i.e.,

$$
\square_{\eta^{\prime}} x^{\prime \alpha} \equiv \frac{1}{\sqrt{-\eta_{0}^{\prime}}} \frac{\partial}{\partial x^{\prime \mu}}\left(\sqrt{-\eta_{0}^{\prime}} \eta^{\prime \mu \nu} \frac{\partial x^{\prime \alpha}}{\partial x^{\prime \nu}}\right)=0,
$$

where $\eta_{\mu \nu}^{\prime}$ is the metric of the reference frame $S_{n}, \eta_{0}^{\prime}=$ Det $\eta_{\mu \nu}^{\prime}$, Det $A_{\mu \nu}$ denotes the value of the corresponding determinant of the tensor $A_{\mu \nu}$; (2) every coordinates $x^{\prime \alpha}$ converges to the Galilean coordinates $(c t, x, y, z)$ at large enough distance, i.e.,

$$
\lim _{r \rightarrow \infty} x^{\prime \alpha}=x^{\alpha}
$$

where $r=\sqrt{x^{2}+y^{2}+z^{2}} ;$ (3) $\eta^{\prime \mu \nu}-\left(\eta^{\prime \mu \nu}\right)_{\infty}$ are outgoing waves, i.e., $\eta^{\prime \mu \nu}-\left(\eta^{\prime \mu \nu}\right)_{\infty}$ satisfy the following condition of outward radiation: for $r \rightarrow \infty$, and all values of $t_{0}^{\prime}=$ $t+r / c$ in an arbitrary fixed interval the following limiting conditions are satisfied ([38], p. 365)

$\lim _{r \rightarrow \infty}\left[\frac{\partial\left[r\left(\eta^{\prime \mu \nu}-\left(\eta^{\prime \mu \nu}\right)_{\infty}\right)\right]}{\partial r}+\frac{1}{c} \frac{\partial\left[r\left(\eta^{\prime \mu \nu}-\left(\eta^{\prime \mu \nu}\right)_{\infty}\right)\right]}{\partial t}\right]=0$

where $\left(\eta^{\prime \mu \nu}\right)_{\infty}$ denotes the value of $\eta^{\prime \mu \nu}$ at infinity. Then, we call this coordinate system $\left(x^{\prime 0}, x^{\prime 1}, x^{\prime 2}, x^{\prime 3}\right)$ as a Fock coordinate system.

We use $S_{F}$ to denote a Fock coordinate system. The Galilean coordinate system $(c t, x, y, z)$ is a Fock coordinate system. V. Fock points out an advantage of Fock coordinate system ([38], p. 369):" When solving Einstein's equations for an isolated system of masses we used harmonic coordinates and in this way obtained a perfectly unambiguous solution." Here the harmonic coordinates called by V. Fock are Fock coordinate systems.

According to a theorem of Fock about Fock coordinate systems ([38], p. 369-373), the transformation equations (34) can be written as a Lorentz transformation, i.e.,

$$
x^{\prime \mu}=a_{\nu}^{\mu} x^{\nu},
$$

where $a_{\nu}^{\mu}$ are coefficients of a Lorentz transformation.

For convenience, we introduce the following notations

$$
\partial_{\mu}^{\prime} \equiv\left(\frac{\partial}{\partial x^{\prime 0}}, \frac{\partial}{\partial x^{\prime 1}}, \frac{\partial}{\partial x^{\prime 2}}, \frac{\partial}{\partial x^{\prime 3}}\right), \quad \partial^{\prime \mu} \equiv \eta^{\prime \mu \nu} \partial_{\nu}^{\prime}
$$

Proposition 3 Suppose that the reference frame $S_{F}$ is a Fock coordinate system and Assumptions 6 is valid, then the total Lagrangian $L_{\mathrm{tot}}^{\prime}$ of a system of the $\Omega(0)$ substratum, the $\Omega(1)$ substratum, vacuum and matter in $S_{F}$ can be written as

$$
\begin{aligned}
L_{\text {tot }}^{\prime}= & \frac{1}{2} \partial_{\lambda}^{\prime} \psi_{\mu \nu}^{\prime} \partial^{\prime \lambda} \psi^{\prime} \mu \nu-2 \partial_{\lambda}^{\prime} \psi_{\mu \nu}^{\prime} \partial^{\prime \mu} \psi^{\prime} \lambda \nu-6 \partial^{\prime} \mu \psi_{\mu \nu}^{\prime} \partial^{\prime} \nu \psi^{\prime} \\
& -\frac{3}{2} \partial_{\lambda}^{\prime} \psi^{\prime} \partial^{\prime \lambda} \psi^{\prime}+L_{\text {more }}^{\prime}+f_{0} \psi_{\mu \nu}^{\prime}\left(T_{m}^{\prime} \mu \nu+T_{\Omega(1)}^{\prime} \mu \nu\right. \\
& +f_{0} \psi_{\mu \nu}^{\text {iner }}\left(T_{m}^{\prime} \mu \nu+T_{\Omega(1)}^{\prime \mu \nu}\right)+O\left[\left(f_{0} \psi_{\mu \nu}^{\text {iner }}\right)^{2}\right] \\
& +O\left[\left(f_{0} \psi_{\mu \nu}^{\prime}\right)^{2}\right]
\end{aligned}
$$

where $\psi_{\mu \nu}^{\prime}$ is a tensorial potential of the gravitational field, $L_{\text {more }}^{\prime}$ denotes those terms involving more than $t$ wo derivatives of $\psi_{\mu \nu}^{\prime}, O\left[\left(f_{0} \psi_{\mu \nu}^{\prime}\right)^{2}\right]$ denotes those terms which are small quantities of the order of $\left(f_{0} \psi_{\mu \nu}^{\prime}\right)^{2}$.

Proof of Proposition 3. Based on some assumptions, the total Lagrangian $L_{\text {tot }}$ of a system of the $\Omega(0)$ substratum, the $\Omega(1)$ substratum and matter in an inertial reference frame can be written as [25]

$$
\begin{aligned}
L_{\text {tot }}= & \frac{1}{2} \partial_{\lambda} \psi_{\mu \nu} \partial^{\lambda} \psi^{\mu \nu}-2 \partial_{\lambda} \psi_{\mu \nu} \partial^{\mu} \psi^{\lambda \nu}-6 \partial^{\mu} \psi_{\mu \nu} \partial^{\nu} \psi \\
& -\frac{3}{2} \partial_{\lambda} \psi \partial^{\lambda} \psi+L_{\text {more }}+f_{0} \psi_{\mu \nu}\left(T_{m}^{\mu \nu}+T_{\Omega(1)}^{\mu \nu}\right) \\
& +O\left[\left(f_{0} \psi_{\mu \nu}\right)^{2}\right]
\end{aligned}
$$

where $\psi_{\mu \nu}$ is a tensorial potential of the gravitational field in the inertial reference frame, $L_{\text {more }}$ denotes those terms involving more than two derivatives of $\psi_{\mu \nu}, O\left[\left(f_{0} \psi_{\mu \nu}\right)^{2}\right]$ denotes those terms which are small quantities of the order of $\left(f_{0} \psi_{\mu \nu}\right)^{2}$.

The total Lagrangian $L_{\text {tot }}^{\prime}$ can be written as

$$
L_{\text {tot }}^{\prime}=L_{\text {tot }}+L_{\text {iner }}
$$

Similar to the case of inertial reference frames ([36], p. $59-60,63)$, we also have the following results in the Fock coordinate system $S_{F}$

$$
\begin{aligned}
& \partial_{\lambda}^{\prime}=a_{\lambda}{ }^{\sigma} \partial_{\sigma}, \quad \partial^{\prime \lambda}=a_{\sigma}^{\lambda} \partial^{\sigma}, \\
& \psi^{\prime \mu \nu}=a_{\alpha}^{\mu} a_{\beta}^{\nu} \psi^{\alpha \beta}, \\
& \psi_{\mu \nu}^{\prime}=a_{\mu}{ }^{\alpha} a_{\nu}{ }^{\beta} \psi_{\alpha \beta} .
\end{aligned}
$$

The first term on the right hand side of Eqs. (40) can be written as

$$
\begin{aligned}
\frac{1}{2} \partial_{\lambda}^{\prime} \psi_{\mu \nu}^{\prime} \partial^{\prime \lambda} \psi^{\prime \mu \nu}= & \frac{1}{2}\left(a_{\lambda}{ }^{\sigma} \partial_{\sigma}\right)\left(a_{\mu}{ }^{\alpha} a_{\nu}{ }^{\beta} \psi_{\alpha \beta}\right) \\
& \cdot\left(a_{\sigma}^{\lambda} \partial^{\sigma}\right)\left(a^{\mu}{ }_{\alpha} a_{\beta}^{\nu} \psi^{\alpha \beta}\right)
\end{aligned}
$$

We have the following result ([36], p. 60)

$$
a_{\beta}^{\mu} a_{\nu}^{\beta}=\delta_{\nu}^{\mu},
$$

where $\delta_{\nu}^{\mu}$ is the Kronecker delta.

Using Eq. (47), Eqs. (46) can be written as

$$
\frac{1}{2} \partial_{\lambda}^{\prime} \psi_{\mu \nu}^{\prime} \partial^{\prime \lambda} \psi^{\prime \mu \nu}=\frac{1}{2} \partial_{\sigma} \psi_{\alpha \beta} \partial^{\sigma} \psi^{\alpha \beta} .
$$


Similarly, we can verify the following results

$$
\begin{aligned}
-2 \partial_{\lambda}^{\prime} \psi_{\mu \nu}^{\prime} \partial^{\prime \mu} \psi^{\prime \lambda \nu} & =-2 \partial_{\sigma} \psi_{\alpha \beta} \partial^{\alpha} \psi^{\sigma \beta}, \\
-6 \partial^{\prime} \mu \psi_{\mu \nu}^{\prime} \partial^{\prime \nu} \psi^{\prime} & =-6 \partial^{\alpha} \psi_{\alpha \beta} \partial^{\beta} \psi, \\
-\frac{3}{2} \partial_{\lambda}^{\prime} \psi^{\prime} \partial^{\prime \lambda} \psi^{\prime} & =-\frac{3}{2} \partial_{\sigma} \psi \partial^{\sigma} \psi, \\
f_{0} \psi_{\mu \nu}^{\prime} T_{m}^{\prime \mu \nu} & =f_{0} \psi_{\alpha \beta} T_{m}^{\alpha \beta}, \\
L_{\text {more }}^{\prime} & =L_{\text {more }} \\
O\left[\left(f_{0} \psi_{\mu \nu}^{\prime}\right)^{2}\right] & =O\left[\left(f_{0} \psi_{\mu \nu}\right)^{2}\right] .
\end{aligned}
$$

Putting Eq. (41) and Eq. (33) into Eq. (42) and using Eqs. (48-54), we obtain Eq. (40).

Applying similar methods as in Ref. [25], we have the following result.

Theorem 1 If we ignore those terms which are smal$l$ quantities of the order of $\left(f_{0} \psi_{\mu \nu}^{\prime}\right)^{2}$ and $\left(f_{0} \psi_{\mu \nu}^{\text {iner }}\right)^{2}$ and those terms involving more than two derivatives of $\psi_{\mu \nu}^{\prime}$ in Eq. (40), i.e., $O\left[\left(f_{0} \psi_{\mu \nu}^{\prime}\right)^{2}\right] \approx 0, O\left[\left(f_{0} \psi_{\mu \nu}^{\text {iner }}\right)^{2}\right] \approx 0$ and $L_{\text {more }}^{\prime} \approx 0$, then the field equations for the total Lagrangian $L_{\text {tot }}^{\prime}$ in Eq. (40) can be written as

$$
\begin{aligned}
& \partial_{\sigma}^{\prime} \partial^{\prime \sigma} \psi_{\alpha \beta}^{\prime}-2\left(\partial^{\prime} \sigma \partial_{\alpha}^{\prime} \psi_{\beta \sigma}^{\prime}+\partial^{\prime} \sigma \partial_{\beta}^{\prime} \psi_{\alpha \sigma}^{\prime}\right) \\
& -6\left(\eta_{\alpha \beta}^{\prime} \partial_{\sigma}^{\prime} \partial_{\lambda}^{\prime} \psi^{\prime \sigma \lambda}+\partial_{\alpha}^{\prime} \partial_{\beta}^{\prime} \psi^{\prime}\right)-3 \eta_{\alpha \beta}^{\prime} \partial_{\sigma}^{\prime} \partial^{\prime \sigma} \psi^{\prime} \\
& =f_{0}\left(T_{\alpha \beta}^{\prime m}+T_{\alpha \beta}^{\prime \Omega(1)}\right) .
\end{aligned}
$$

Proof of Theorem 1. We have the following EulerLagrange equations [41]

$$
\frac{\partial L_{\text {tot }}^{\prime}}{\partial \psi^{\prime \alpha \beta}}-\frac{\partial}{\partial x^{\prime} \sigma}\left(\frac{\partial L_{\text {tot }}^{\prime}}{\partial\left(\partial_{\sigma}^{\prime} \psi^{\prime} \alpha \beta\right)}\right)=0 .
$$

We have the following results

$$
\begin{gathered}
\frac{\partial\left(\partial_{\lambda}^{\prime} \psi_{\mu \nu}^{\prime} \partial^{\prime \lambda} \psi^{\prime} \mu \nu\right)}{\partial\left(\partial_{\sigma}^{\prime} \psi^{\prime \alpha \beta}\right)}=\frac{\partial\left(\partial_{\lambda}^{\prime} \psi_{\mu \nu}^{\prime}\right)}{\partial\left(\partial_{\sigma}^{\prime} \psi^{\prime} \alpha \beta\right)} \partial^{\prime \lambda} \psi^{\prime} \mu \nu \\
+\partial_{\lambda}^{\prime} \psi_{\mu \nu}^{\prime} \frac{\partial\left(\partial^{\prime} \lambda \psi^{\prime} \mu \nu\right)}{\partial\left(\partial_{\sigma}^{\prime} \psi^{\prime \alpha \beta}\right)} \\
\psi_{\mu \nu}^{\prime}=\eta_{\mu \rho}^{\prime} \eta_{\nu \tau}^{\prime} \psi^{\prime} \rho \tau
\end{gathered}
$$

Using Eqs. (58), we have

$$
\begin{aligned}
& \frac{\partial\left(\partial_{\lambda}^{\prime} \psi_{\mu \nu}^{\prime}\right)}{\partial\left(\partial_{\sigma}^{\prime} \psi^{\prime} \alpha \beta\right)}=\frac{\partial}{\partial\left(\partial_{\sigma}^{\prime} \psi^{\prime} \alpha \beta\right)}\left(\eta_{\mu \rho}^{\prime} \eta_{\nu \tau}^{\prime} \partial_{\lambda}^{\prime} \psi^{\prime} \rho \tau\right) \\
& =\eta_{\mu \rho}^{\prime} \eta_{\nu \tau}^{\prime} \frac{\partial\left(\partial_{\lambda}^{\prime} \psi^{\prime} \rho \tau\right)}{\partial\left(\partial_{\sigma}^{\prime} \psi^{\prime \alpha \beta}\right)} \\
& =\eta_{\mu \rho}^{\prime} \eta_{\nu \tau}^{\prime} \delta_{\sigma}^{\lambda} \delta_{\alpha}^{\rho} \delta_{\beta}^{\tau} \\
& =\eta_{\mu \alpha}^{\prime} \eta_{\nu \beta}^{\prime} \delta_{\sigma}^{\lambda} \text {. }
\end{aligned}
$$

Using Eqs. (59) and Eqs. (58), the first term on the right hand side of Eqs. (57) can be written as

$$
\frac{\partial\left(\partial_{\lambda}^{\prime} \psi_{\mu \nu}^{\prime}\right)}{\partial\left(\partial_{\sigma}^{\prime} \psi^{\prime \alpha \beta}\right)} \partial^{\prime \lambda} \psi^{\prime} \mu \nu=\partial^{\prime} \sigma \psi_{\alpha \beta}^{\prime} .
$$

Similarly, the second term on the right hand side of Eqs. (57) can be written as

$$
\left.\partial_{\lambda}^{\prime} \psi_{\mu \nu}^{\prime} \frac{\partial\left(\partial^{\prime} \lambda \psi^{\prime} \mu \nu\right)}{\partial\left(\partial_{\sigma}^{\prime} \psi^{\prime} \alpha \beta\right.}\right)=\partial^{\prime \sigma} \psi_{\alpha \beta}^{\prime} .
$$

Using Eqs. (57), Eqs. (60) and Eqs. (61), we have

$$
\frac{\partial}{\partial x^{\prime \sigma}}\left[\frac{\partial\left(\partial_{\lambda}^{\prime} \psi_{\mu \nu}^{\prime} \partial^{\prime} \lambda \psi^{\prime} \mu \nu\right.}{\partial\left(\partial_{\sigma}^{\prime} \psi^{\prime \alpha \beta}\right)}\right]=2 \partial_{\sigma}^{\prime} \partial^{\prime} \sigma \psi_{\alpha \beta}^{\prime} .
$$

Similarly, we can verify the following results

$$
\begin{aligned}
\frac{\partial}{\partial x^{\prime} \sigma}\left[\frac{\partial\left(\partial_{\lambda}^{\prime} \psi_{\mu \nu}^{\prime} \partial^{\prime} \mu \psi^{\prime} \lambda \nu\right)}{\partial\left(\partial_{\sigma}^{\prime} \psi^{\prime \alpha \beta}\right)}\right] & =\partial^{\prime} \sigma \partial_{\alpha}^{\prime} \psi_{\beta \sigma}^{\prime} \\
& +\partial^{\prime} \sigma \partial_{\beta}^{\prime} \psi_{\alpha \sigma}^{\prime} \\
\frac{\partial}{\partial x^{\prime} \sigma}\left[\frac{\partial\left(\partial^{\prime} \mu \psi_{\mu \nu}^{\prime} \partial^{\prime} \nu\right)}{\partial\left(\partial_{\sigma}^{\prime} \psi^{\prime \alpha \beta}\right)}\right] & =\partial_{\alpha}^{\prime} \partial_{\beta}^{\prime} \psi^{\prime} \\
& +\eta_{\alpha \beta}^{\prime} \partial_{\sigma}^{\prime} \partial_{\lambda}^{\prime} \psi^{\prime} \sigma \lambda \\
\frac{\partial}{\partial x^{\prime} \sigma}\left[\frac{\partial\left(\partial_{\lambda}^{\prime} \psi^{\prime} \partial^{\prime} \lambda \psi^{\prime}\right)}{\partial\left(\partial_{\sigma}^{\prime} \psi^{\prime} \alpha \beta\right.}\right] & =2 \eta_{\alpha \beta}^{\prime} \partial_{\sigma}^{\prime} \partial^{\prime} \sigma \psi^{\prime} \\
\frac{\partial L_{\mathrm{tot}}^{\prime}}{\partial \psi^{\prime} \alpha \beta} & =f_{0}\left(T_{\alpha \beta}^{\prime} m+T_{\alpha \beta}^{\prime \Omega(1)}\right) .
\end{aligned}
$$

Putting Eq. (40) into Eqs. (56) and using Eqs. (62-66), we obtain Eqs. (55).

Following Ref. [25], we introduce the following notation in the Fock coordinate system $S_{F}$.

$$
\begin{aligned}
& \Psi^{\prime} \mu \nu=\partial_{\lambda}^{\prime} \partial^{\prime} \lambda \psi^{\prime} \mu \nu-2 \partial_{\lambda}^{\prime} \partial^{\prime} \mu \psi^{\prime} \nu \lambda-2 \partial_{\lambda}^{\prime} \partial^{\prime} \nu \psi^{\prime} \mu \lambda \\
& -6 \eta^{\prime \mu \nu} \partial_{\sigma}^{\prime} \partial_{\lambda}^{\prime} \psi^{\prime \sigma \lambda}-6 \partial^{\prime} \mu \partial^{\prime} \nu \psi^{\prime}-3 \eta^{\prime \mu \nu} \partial_{\lambda}^{\prime} \partial^{\prime \lambda} \psi^{\prime} \text {. }
\end{aligned}
$$

For convenience, we introduce the following definition of the total energy-momentum tensor $T^{\prime} \mu \nu$ of the system of the matter, the $\Omega(1)$ substratum and the $\Omega(0)$ substratum in the Fock coordinate system $S_{F}$

$$
T^{\prime \mu \nu}=T_{m}^{\prime \mu \nu}+T_{\Omega(1)}^{\prime \mu \nu}+T_{\Omega(0)}^{\prime \mu \nu},
$$

where $T_{\Omega(0)}^{\prime} \mu \nu$ is the energy-momentum tensor of the $\Omega(0)$ substratum in the Fock coordinate system $S_{F}$.

We introduce the following notation in $S_{F}$

$$
\begin{aligned}
& \Theta^{\prime} \mu \nu=\partial_{\lambda}^{\prime} \partial^{\prime} \lambda \psi^{\prime} \mu \nu-\left(\partial_{\lambda}^{\prime} \partial^{\prime} \mu \psi^{\prime} \nu \lambda+\partial_{\lambda}^{\prime} \partial^{\prime} \nu \psi^{\prime} \mu \lambda\right) \\
& +\left(\partial^{\prime \mu} \partial^{\prime \nu} \psi^{\prime}+\eta^{\prime \mu \nu} \partial_{\sigma}^{\prime} \partial_{\lambda}^{\prime} \psi^{\prime} \sigma \lambda\right)-\eta^{\prime \mu \nu} \partial_{\lambda}^{\prime} \partial^{\prime \lambda} \psi^{\prime} .
\end{aligned}
$$

Following Ref. [25], we introduce the following definition of the contravariant energy-momentum tensor $T_{\omega}^{\prime} \mu \nu$ of vacuum in $S_{F}$

$$
T_{\omega}^{\prime} \mu \nu=\frac{1}{f_{0}} \Psi^{\prime} \mu \nu+\frac{1}{f_{0}} \Theta^{\prime} \mu \nu+T_{\Omega(0)}^{\prime} \mu \nu .
$$

Using these definitions, we have the following result. 
Corollary 1 The field equations (55) can be written as

$$
\begin{aligned}
& \partial_{\lambda}^{\prime} \partial^{\prime} \lambda \psi^{\prime} \mu \nu-\partial_{\lambda}^{\prime} \partial^{\prime} \mu \psi^{\prime} \nu \lambda-\partial_{\lambda}^{\prime} \partial^{\prime \nu} \psi^{\prime} \mu \lambda \\
& +\partial^{\prime} \mu \partial^{\prime} \nu \psi^{\prime}+\eta^{\prime \mu \nu} \partial_{\sigma}^{\prime} \partial_{\lambda}^{\prime} \psi^{\prime} \sigma \lambda \\
& -\eta^{\prime} \mu \nu \partial_{\lambda}^{\prime} \partial^{\prime} \lambda \psi^{\prime}=-f_{0}\left(T^{\prime} \mu \nu-T_{\omega}^{\prime} \mu \nu\right)
\end{aligned}
$$

We can verify that the field equations (71) are invariant under the following gauge transformation

$$
\psi^{\prime \mu \nu} \rightarrow \psi^{\prime \mu \nu}+\partial^{\prime \mu} \Lambda^{\nu}+\partial^{\prime} \nu \Lambda^{\mu},
$$

where $\Lambda^{\mu}$ is an arbitrary vector field.

We introduce the following definition

$$
\phi^{\prime \mu \nu}=\psi^{\prime} \mu \nu-\frac{1}{2} \eta^{\prime \mu \nu} \psi^{\prime} .
$$

Using Eqs. (73), the field equations (71) can be written as

$$
\begin{aligned}
& \partial_{\lambda}^{\prime} \partial^{\prime \lambda} \phi^{\prime \mu \nu}-\partial_{\lambda}^{\prime} \partial^{\prime \mu} \phi^{\prime \nu \lambda}-\partial_{\lambda}^{\prime} \partial^{\prime \nu} \phi^{\mu \lambda} \\
& +\eta^{\prime \mu \nu} \partial_{\sigma}^{\prime} \partial_{\lambda}^{\prime} \phi^{\prime} \sigma \lambda=-f_{0}\left(T^{\prime \mu \nu}-T_{\omega}^{\prime \mu \nu}\right) .
\end{aligned}
$$

We introduce the following Hilbert gauge condition [39]

$$
\partial_{\mu}^{\prime}\left(\psi^{\prime} \mu \nu-\frac{1}{2} \eta^{\prime \mu \nu} \psi^{\prime}\right)=0
$$

Using Eqs. (73), the Hilbert gauge condition Eqs. (75) simplifies to

$$
\partial_{\mu}^{\prime} \phi^{\prime \mu \nu}=0
$$

If we impose the Hilbert gauge condition Eqs. (76) on the fields, then the field equations (74) simplify to

$$
\partial_{\lambda}^{\prime} \partial^{\prime \lambda} \phi^{\prime \mu \nu}=-f_{0}\left(T^{\prime \mu \nu}-T_{\omega}^{\prime} \mu \nu\right) .
$$

The field equations (77) can also be written as

$$
\eta^{\prime \alpha \beta} \frac{\partial^{2} \phi^{\prime} \mu \nu}{\partial x^{\prime \alpha} \partial x^{\prime \beta}}=-f_{0}\left(T^{\prime \mu \nu}-T_{\omega}^{\prime \mu \nu}\right)
$$

\section{GENERALIZED EINSTEIN'S EQUATIONS IN A SPECIAL CLASS OF NON-INERTIAL REFERENCE FRAMES}

Definition 5 The Einstein tensor $G_{\mu \nu}$ is defined by

$$
G_{\mu \nu} \equiv R_{\mu \nu}-\frac{1}{2} g_{\mu \nu} R
$$

where $g_{\mu \nu}$ is a metric tensor of a Riemannian spacetime, $R_{\mu \nu}$ is the Ricci tensor, $R \equiv g^{\mu \nu} R_{\mu \nu}, g^{\mu \nu}$ is the corresponding contravariant tensor of $g_{\mu \nu}$ such that $g_{\mu \lambda} g^{\lambda \nu}=\delta_{\mu}^{\nu}([37], p .40)$.

Similar to Ref. [25], we introduce the following definition of a metric tensor $g_{\mu \nu}$ of a Riemannian spacetime.

\section{Definition 6}

$$
\tilde{g}^{\mu \nu} \equiv \sqrt{-g_{0}} g^{\mu \nu} \equiv \eta^{\prime \mu \nu}-2 f_{0} \phi^{\prime \mu \nu},
$$

where $g_{0}=$ Det $g_{\mu \nu}$.

Applying similar methods of V. Fock ([38], p. 422$430)$, Fock's theorem of the Einstein tensor $G_{\mu \nu}$ in the Galilean coordinates ([38], p. 429) can be generalized to non-inertial coordinate systems $\left(x^{\prime 0}, x^{\prime 1}, x^{\prime 2}, x^{\prime 3}\right)$.

Proposition 4 The contravariant Einstein tensor $G^{\mu \nu}$ in a non-inertial coordinate systems $\left(x^{\prime 0}, x^{\prime 1}, x^{\prime 2}, x^{\prime 3}\right)$ can be written as

$$
\begin{aligned}
G^{\mu \nu}= & \frac{1}{2 g_{0}} \tilde{g}^{\alpha \beta} \frac{\partial^{2} \tilde{g}^{\mu \nu}}{\partial x^{\prime} \alpha \partial x^{\prime \beta}}+\Pi^{\prime \mu, \alpha \beta} \Pi_{\alpha \beta}^{\prime}-\frac{1}{2} y^{\prime \mu} y^{\prime}{ }^{\prime} \\
& +\frac{1}{2} g^{\mu \nu}\left(L^{\prime}+B^{\prime}\right)-B^{\prime} \mu \nu
\end{aligned}
$$

where

$$
\Pi^{\prime \mu, \alpha \beta} \equiv \frac{1}{2 g_{0}}\left(\tilde{g}^{\alpha \lambda} \frac{\partial \tilde{g}^{\mu \beta}}{\partial x^{\prime \lambda}}+\tilde{g}^{\beta \lambda} \frac{\partial \tilde{g}^{\mu \alpha}}{\partial x^{\prime \lambda}}-\tilde{g}^{\mu \lambda} \frac{\partial \tilde{g}^{\alpha \beta}}{\partial x^{\prime \lambda}}\right),
$$

$$
\Pi_{\alpha \beta}^{\prime}{ }^{\prime} \equiv g_{\alpha \lambda} g_{\beta \sigma} \Pi^{\prime} \nu, \lambda \sigma
$$

$$
\Gamma^{\prime \alpha} \equiv g^{\sigma \lambda} \Gamma_{\sigma \lambda}^{\prime \alpha}
$$

$$
\Gamma_{\alpha \beta}^{\prime \nu} \equiv \frac{1}{2} g^{\mu \nu}\left(\frac{\partial g_{\mu \alpha}}{\partial x^{\prime \beta}}+\frac{\partial g_{\mu \beta}}{\partial x^{\prime \alpha}}-\frac{\partial g_{\alpha \beta}}{\partial x^{\prime \mu}}\right)
$$

$$
\begin{gathered}
\Gamma^{\prime \mu \nu} \equiv \frac{1}{2}\left(g^{\mu \alpha} \frac{\partial \Gamma^{\prime \nu}}{\partial x^{\prime \alpha}}+g^{\nu \alpha} \frac{\partial \Gamma^{\prime \mu}}{\partial x^{\prime \alpha}}-\frac{\partial g^{\mu \nu}}{\partial x^{\prime \alpha}} \Gamma^{\prime \alpha}\right), \\
y_{\beta}^{\prime} \equiv \frac{\partial\left(\lg \sqrt{-g_{0}}\right)}{\partial x^{\prime \beta}}, y^{\prime \alpha} \equiv g^{\alpha \beta} y_{\beta}^{\prime},
\end{gathered}
$$

$$
L^{\prime} \equiv-\frac{1}{2 \sqrt{-g_{0}}} \Gamma_{\alpha \beta}^{\prime}{ }^{\nu} \frac{\partial \tilde{g}^{\alpha \beta}}{\partial x^{\prime \nu}}+\frac{1}{2} y_{\nu}^{\prime} y^{\prime \nu}
$$

$B^{\prime \mu \nu} \equiv \Gamma^{\prime \mu \nu}+\frac{1}{2}\left(y^{\prime} \mu \Gamma^{\prime} \nu+y^{\prime} \Gamma^{\prime} \mu\right), B^{\prime} \equiv g_{\mu \nu} B^{\prime \mu \nu}$.

A proof of Proposition 4 can be found in the Appendix.

Theorem 2 In a Fock coordinate system $S_{F}$, we have the following field equations

$$
\begin{aligned}
& G^{\mu \nu}-\frac{1}{2 g_{0}}\left(\sqrt{-g_{0}} g^{\alpha \beta}-\eta^{\prime \alpha \beta}\right) \frac{\partial^{2}\left(\sqrt{-g_{0}} g^{\mu \nu}\right)}{\partial x^{\prime \alpha} \partial x^{\prime \beta}} \\
& -\frac{1}{2 g_{0}} \eta^{\prime \alpha \beta} \frac{\partial^{2} \eta^{\prime} \mu \nu}{\partial x^{\prime \alpha} \partial x^{\prime \beta}}-\Pi^{\prime \mu, \alpha \beta} \Pi_{\alpha \beta}^{\prime}{ }^{\prime}+\frac{1}{2} y^{\prime \mu} y^{\prime}{ }^{\prime} \\
& -\frac{1}{2} g^{\mu \nu}\left(L^{\prime}+B^{\prime}\right)+B^{\prime} \mu \nu=\frac{f_{0}^{2}}{g_{0}}\left(T^{\prime} \mu \nu-T_{\omega}^{\prime}{ }^{\mu \nu}\right) .
\end{aligned}
$$


Proof of Theorem 2. Using Eqs. (80), Eqs. (81) can be written as

$$
\begin{aligned}
& G^{\mu \nu}=\frac{1}{2 g_{0}}\left(\sqrt{-g_{0}} g^{\alpha \beta}-\eta^{\prime \alpha \beta}+\eta^{\prime \alpha \beta}\right) \frac{\partial^{2}\left(\eta^{\prime \mu \nu}-2 f_{0} \phi^{\prime \mu \nu}\right)}{\partial x^{\prime \alpha} \partial x^{\prime \beta}} \\
& +\Pi^{\prime}{ }^{\mu, \alpha \beta} \Pi_{\alpha \beta}^{\prime}{ }^{\nu}-\frac{1}{2} y^{\prime}{ }^{\prime} y^{\prime \nu}+\frac{1}{2} g^{\mu \nu}\left(L^{\prime}+B^{\prime}\right)-B^{\prime} \mu \nu
\end{aligned}
$$

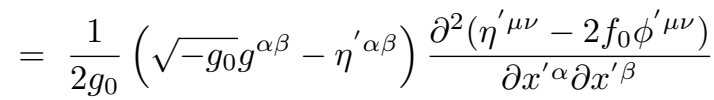

$$
\begin{aligned}
& +\frac{1}{2 g_{0}} \eta^{\prime \alpha \beta} \frac{\partial^{2} \eta^{\prime \mu \nu}}{\partial x^{\prime \alpha} \partial x^{\prime \beta}}-\frac{f_{0}}{g_{0}} \eta^{\prime \alpha \beta} \frac{\partial^{2} \phi^{\prime} \mu \nu}{\partial x^{\prime \alpha} \partial x^{\prime \beta}} \\
& +\Pi^{\prime \mu, \alpha \beta} \Pi_{\alpha \beta}^{\prime \nu}-\frac{1}{2} y^{\prime}{ }^{\prime} y^{\prime \nu} \\
& +\frac{1}{2} g^{\mu \nu}\left(L^{\prime}+B^{\prime}\right)-B^{\prime \mu \nu} .
\end{aligned}
$$

Using Eqs. (80) and Eqs. (78), Eqs. (91) can be written as Eqs. (90).

We need to study the relationships between Eqs. (90) and the Einstein's field equations. In a harmonic coordinates system, we have ([38], p. 254)

$$
\Gamma^{\prime \nu}=\Gamma^{\prime \mu \nu}=B^{\prime \mu \nu}=B^{\prime}=0 .
$$

Using Eqs. (92) and Eqs. (90), we have the following result.

Corollary 2 In a Fock coordinate system $S_{F}$ the field equations (90) can be written as

$$
\begin{aligned}
G^{\mu \nu} & -\frac{1}{2 g_{0}}\left(\sqrt{-g_{0}} g^{\alpha \beta}-\eta^{\prime \alpha \beta}\right) \frac{\partial^{2}\left(\sqrt{-g_{0}} g^{\mu \nu}\right)}{\partial x^{\prime \alpha} \partial x^{\prime \beta}} \\
& -\frac{1}{2 g_{0}} \eta^{\prime \alpha \beta} \frac{\partial^{2} \eta^{\prime} \mu \nu}{\partial x^{\prime \alpha} \partial x^{\prime \beta}}-\Pi^{\prime \mu, \alpha \beta} \Pi_{\alpha \beta}^{\prime}{ }^{\prime} \\
& +\frac{1}{2} y^{\prime} y^{\prime}{ }^{\nu}-\frac{1}{2} g^{\mu \nu} L^{\prime}=\frac{f_{0}^{2}}{g_{0}}\left(T^{\prime} \mu \nu-T_{\omega}^{\prime \mu \nu}\right) .
\end{aligned}
$$

Definition 7 If the following conditions are valid

$$
\begin{aligned}
& \eta^{\prime \mu \nu} \approx \eta^{\mu \nu}, \\
& \left|\frac{1}{2} \eta^{\prime \alpha \beta} \frac{\partial^{2} \eta^{\prime} \mu \nu}{\partial x^{\prime \alpha} \partial x^{\prime \beta}}\right| \ll\left|f_{0}^{2}\left(T^{\prime} \mu \nu-T_{\omega}^{\prime} \mu \nu\right)\right|,
\end{aligned}
$$

then we call this reference frame quasi-inertial.

Using Eqs. (95) and Eqs. (93), we have the following result.

Corollary 3 If the reference frame $S_{F}$ is quasi-inertial, then, the field equations (93) can be written as

$$
\begin{aligned}
& G^{\mu \nu}-\frac{1}{2 g_{0}}\left(\sqrt{-g_{0}} g^{\alpha \beta}-\eta^{\prime \alpha \beta}\right) \frac{\partial^{2}\left(\sqrt{-g_{0}} g^{\mu \nu}\right)}{\partial x^{\prime \alpha} \partial x^{\prime \beta}} \\
& -\Pi^{\prime}{ }^{\mu, \alpha \beta} \Pi_{\alpha \beta}^{\prime}{ }^{\nu}+\frac{1}{2} y^{\prime}{ }^{\prime} y^{\prime}{ }^{\nu}-\frac{1}{2} g^{\mu \nu} L^{\prime} \\
& \approx \frac{f_{0}^{2}}{g_{0}}\left(T^{\prime}{ }^{\mu \nu}-T_{\omega}^{\prime} \mu \nu\right) .
\end{aligned}
$$

Eqs. (96) are only valid approximately in a quasiinertial Fock coordinate system $S_{F}$. Now we consider weak fields.

Definition 8 If $\phi^{\prime \mu \nu}$ and their first and higher derivatives satisfy the following conditions

$$
\begin{aligned}
& \left|2 f_{0} \phi^{\prime} \mu \nu\right| \ll 1 \\
& \left|\frac{\partial^{j+k}\left(2 f_{0} \phi^{\prime} \mu \nu\right.}{\partial\left(x^{\prime \alpha}\right)^{j} \partial\left(x^{\prime \beta}\right)^{k}}\right| \ll 1, j+k=1,2,3, \cdots
\end{aligned}
$$

then we call this field $\phi^{\prime \mu \nu}$ weak.

Following similar ideas as in Ref. [25], we introduce the following assumption.

Assumption 7 For weak fields, the following relationships are valid

$$
T^{\prime \mu \nu}-T_{\omega}^{\prime \mu \nu} \approx T_{m}^{\prime \mu \nu}+T_{\Omega(1)}^{\prime \mu \nu} .
$$

Similar to Ref. [25], we have the following result.

Corollary 4 Suppose that (1) the Fock coordinate system $S_{F}$ is quasi-inertial; (2) the field is weak. Then, the field equations (96) reduce to

$$
R_{\mu \nu}-\frac{1}{2} g_{\mu \nu} R \approx \frac{f_{0}^{2}}{g_{0}}\left(T_{\mu \nu}^{\prime m}+T_{\mu \nu}^{\prime \Omega(1)}\right) .
$$

Proof of Corollary 4. According to Definition 8, $f_{0} \phi^{\prime \mu \nu}$ and their first and higher derivatives are small quantities of order $\varepsilon$, where $|\varepsilon| \ll 1$ is a small quantity. Since the reference frame is quasi-inertial, Eqs. (94) are valid. Using Eqs. (94), Eqs. (80) can be written as

$$
\sqrt{-g_{0}} g^{\mu \nu} \approx \eta^{\mu \nu}-2 f_{0} \phi^{\prime \mu \nu} .
$$

Since the field is weak, Eqs. (97) and Eqs. (98) are valid. Thus, applying Eqs. (97) and Eqs. (101), we have the following estimations of the order of magnitude of the following quantities

$$
\sqrt{-g_{0}} g^{\alpha \beta}-\eta^{\prime \alpha \beta} \sim \varepsilon .
$$

Using Eqs. (98), we have the following estimations

$$
\frac{\partial g_{\mu \nu}}{\partial x^{\prime \alpha}} \sim \frac{\partial g^{\mu \nu}}{\partial x^{\prime \alpha}} \sim \varepsilon
$$

Applying Eqs. (101) and Eqs. (98), we have the following estimations

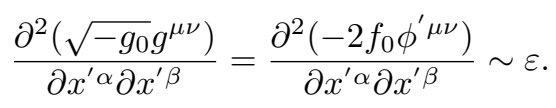

Thus, using Eqs. (102) and Eqs. (104), we have the following estimations

$$
\left(\sqrt{-g_{0}} g^{\alpha \beta}-\eta^{\prime \alpha \beta}\right) \frac{\partial^{2}\left(\sqrt{-g_{0}} g^{\mu \nu}\right)}{\partial x^{\prime \alpha} \partial x^{\prime \beta}} \sim \varepsilon^{2} .
$$


Applying Eqs. (82), Eqs. (83) and Eqs. (103), we have the following estimations

$$
\Pi^{\prime \mu, \alpha \beta} \sim \Pi_{\alpha \beta}^{\prime \nu} \sim \varepsilon
$$

Using Eqs. (87), we have the following relationship $([38]$, p. 143)

$$
y_{\beta}^{\prime}=\Gamma_{\beta \nu}^{\prime \nu} .
$$

We also have ([38], p. 143)

$$
\Gamma_{\beta \nu}^{\prime \nu}=\frac{1}{2} g^{\mu \nu} \frac{\partial g_{\mu \nu}}{\partial x^{\prime \beta}} .
$$

Applying Eqs. (107), Eqs. (108) and Eqs. (103), we have the following estimations

$$
y_{\beta}^{\prime} \sim \varepsilon .
$$

Using Eqs. (87) and Eqs. (109), we have the following estimations

$$
y^{\prime \alpha} \sim \varepsilon
$$

Similar to the case of the Galilean coordinates, we have ([38], p. 430)

$$
L^{\prime}=-\frac{1}{2} \Gamma_{\alpha \beta}^{\prime \nu} \frac{\partial g^{\alpha \beta}}{\partial x^{\prime \nu}}-\Gamma^{\prime \alpha} y_{\alpha}^{\prime} .
$$

Applying Eqs. (92), Eq. (111) can be written as

$$
L^{\prime}=-\frac{1}{2} \Gamma_{\alpha \beta}^{\prime \nu} \frac{\partial g^{\alpha \beta}}{\partial x^{\prime \nu}} .
$$

Using Eq. (112), Eqs. (85) and Eqs. (103), we have the following estimation

$$
L^{\prime} \sim \varepsilon^{2}
$$

Applying Eqs. (105), Eqs. (106), Eqs. (110) and Eq. (113), we see that the second to the fifth term on the left side of Eqs. (96) are all small quantities of order $\varepsilon^{2}$. Ignoring all these small quantities of order $\varepsilon^{2}$ in Eqs. (96) and using Eqs. (99), we obtain

$$
G^{\mu \nu} \approx \frac{f_{0}^{2}}{g_{0}}\left(T_{m}^{\prime \mu \nu}+T_{\Omega(1)}^{\prime \mu \nu}\right) .
$$

Applying the rules of lowering the indexes of tensors, i.e., $G^{\mu \nu}=g^{\mu \sigma} g^{\nu \lambda} G_{\sigma \lambda}, T_{m}^{\prime \mu \nu}=g^{\mu \sigma} g^{\nu \lambda} T_{\sigma \lambda}^{\prime m}, T_{\Omega(1)}^{\prime \mu \nu}=$ $g^{\mu \sigma} g^{\nu \lambda} T_{\sigma \lambda}^{\prime} \Omega(1)$, Eqs. (114) can be written as

$$
G_{\sigma \lambda} \approx \frac{f_{0}^{2}}{g_{0}}\left(T_{\sigma \lambda}^{\prime m}+T_{\sigma \lambda}^{\prime} \Omega(1)\right) .
$$

Putting Eqs. (79) into Eqs. (115), we obtain Eqs. (100).

Using Eq. (23), the field equations (100) can be written as

$$
R_{\mu \nu}-\frac{1}{2} g_{\mu \nu} R \approx \frac{1}{g_{0}} \frac{8 \pi \gamma_{N}}{c^{4}}\left(T_{\mu \nu}^{\prime m}+T_{\mu \nu}^{\prime \Omega(1)}\right) .
$$

Similar to Ref. [25], we have the following result.
Corollary 5 Suppose that (1) the Fock coordinate system $S_{F}$ is quasi-inertial; (2) the field is weak; (3) $g_{0} \approx$ -1 . Then, the field equations (100) reduce to

$$
R_{\mu \nu}-\frac{1}{2} g_{\mu \nu} R \approx-f_{0}^{2}\left(T_{\mu \nu}^{\prime} m+T_{\mu \nu}^{\prime} \Omega(1)\right)
$$

We introduce the following notation

$$
\kappa=f_{0}^{2}=\frac{8 \pi \gamma_{N}}{c^{4}}
$$

Using Eq. (118), the field equations (100) can be written as

$$
R_{\mu \nu}-\frac{1}{2} g_{\mu \nu} R \approx \frac{\kappa}{g_{0}}\left(T_{\mu \nu}^{\prime m}+T_{\mu \nu}^{\prime \Omega(1)}\right) .
$$

Using Eq. (118), the field equations (117) can be written as

$$
R_{\mu \nu}-\frac{1}{2} g_{\mu \nu} R \approx-\kappa\left(T_{\mu \nu}^{\prime m}+T_{\mu \nu}^{\prime \Omega(1)}\right) .
$$

We notice that the field equations (120) are the Einstein's equations [1-3] if we suppose that $T_{\mu \nu}^{\prime \Omega(1)} \approx 0$. Therefore, the field equations (90) are generalizations of the Einstein's equations in some special non-inertial reference frames. Thus, all known experiments of gravitational phenomena which support the theory of general relativity may also be explained by this theory of gravity based on the theory of vacuum mechanics [25-28].

\section{DISCUSSION}

Although the field equations (90) are generalizations of the Einstein's equations, there exist at least the following differences between this theory and the theory of general relativity.

1. In the theory of general relativity, the Einstein's equations are assumptions [1-3]. Although A. Einstein introduced his new concept of gravitational aether ([17], p. 63-113), he did not derive his equations theoretically based on his new concept of the gravitational aether. In our theory, the generalized Einstein's equations (90) are derived by methods of special relativistic continuum mechanics based on some assumptions.

2. Although the theory of general relativity is a field theory of gravity, the definitions of gravitational fields are not based on continuum mechanics [1-3, 42]. Because of the absence of a continuum, the theory of general relativity may be regarded as a phenomenological theory of gravity. In our theory, gravity is transmitted by the $\Omega(0)$ substratum. The tensorial potential $\psi_{\mu \nu}^{\prime}$ of gravitational fields are defined based on special relativistic continuum mechanics.

3. In Einstein's theory, the concept of Riemannian spacetime is introduced together with the field equations [1-3]. The theory of general relativity cannot provide a 
physical definition of the metric tensor of the Riemannian spacetime. In our theory, the background spacetime is the Minkowski spacetime. However, the initial flat background spacetime is no longer physically observable. According to the equation of motion of a point particle in gravitational field in inertial reference frames [25], to the first order of $f_{0} \psi_{\mu \nu}$, the physically observable spacetime is a Riemannian spacetime with the metric tensor $g_{\mu \nu}$. The metric tensor $g_{\mu \nu}$ is defined based on the tensorial potential $\psi_{\mu \nu}^{\prime}$ of gravitational fields.

4. The masses of particles are constants in the theory of general relativity [1-3]. In our theory, the masses of particles are functions of time $t$ [27].

5. The gravitational constant $G_{N}$ is a constant in the theory of general relativity [1-3]. The theory of general relativity cannot provide a derivation of $G_{N}$. In our theory, the parameter $\gamma_{N}$ is derived theoretically. From Eq. (4), we see that $\gamma_{N}$ depends on time $t$.

6. In our theory, the parameter $\gamma_{N}$ in Eq. (4) depends on the density $\rho_{0}$ of the $\Omega(0)$ substratum. If $\rho_{0}$ varies from place to place, i.e., $\rho_{0}=\rho_{0}(t, x, y, z)$, then the space dependence of the gravitational constant $\gamma_{N}$ can be seen from Eq. (4).

7. The Einstein's equations [1-3] are supposed to be valid in all reference frames. It is known that general relativity is a pure theory and contains no adjustable constants. So the predictions of general relativity are fixed. Thus, every experimental test of the theory is important. For more than 100 years, experimental tests of general relativity are carried out only in the solar system $[43,44]$. However, the solar system can be regarded approximately as an inertial reference frame. Therefore, it is still not clear whether the Einstein's equations are valid in all non-inertial reference frames or not. However, in our theory the generalized Einstein's equations (90) are valid only in Fock coordinate systems. Whether it is possible for us to derive generalized Einstein's equations in other non-inertial reference frames is an interesting question. V. Fock said ([38], p. 394):" But physically the 'general principle of relativity', in the sense that corresponding processes exist in arbitrary reference frames, does not hold at all. Therefore Einstein's conclusion that all reference frames are physically equivalent, is without foundation."

8. The Einstein's equations are rigorous [1-3]. However, in our theory, Eqs. (120) are valid approximately under some assumptions.

It is interesting whether it is possible for us to detect some of these differences by experiments.

\section{CONCLUSION}

Inspired by the mathematical forms of the dynamical gravitational potentials in inertial reference frames, we establish mathematical models of inertial potential and inertial force Lagrangian in non-inertial reference frames. Field equations of gravitation in Fock coordinate systems are derived based on the Euler-Lagrange equations. Applying the generalized Fock's theorem, generalized Einstein's equations in Fock coordinate systems are derived. If the field is weak and the reference frame is quasiinertial, these generalized Einstein's equations reduce to Einstein's equations.

\section{Acknowledgments}

This work was partly supported by the Doctor Research Foundation of Henan Polytechnic University (Grant No. B2012-069). I would like to thank an anonymous reviewer for his important remarks and opinions.

\section{Appendix}

Proof of Proposition 4. The definition of the covariant second rank curvature tensor $R_{\mu \nu}$ is ([38], p. 422)

$$
R_{\mu \nu} \equiv g^{\alpha \beta} R_{\mu \alpha, \beta \nu}
$$

where

$$
\begin{aligned}
R_{\mu \alpha, \beta \nu} \equiv & \frac{1}{2}\left(\frac{\partial^{2} g_{\mu \nu}}{\partial x^{\prime \alpha} \partial x^{\prime \beta}}+\frac{\partial^{2} g_{\alpha \beta}}{\partial x^{\prime} \mu \partial x^{\prime \nu}}-\frac{\partial^{2} g_{\nu \alpha}}{\partial x^{\prime} \mu \partial x^{\prime \beta}}\right. \\
& \left.-\frac{\partial^{2} g_{\mu \beta}}{\partial x^{\prime} \nu \partial x^{\prime \alpha}}\right)-g_{\sigma \lambda} \Gamma_{\mu \beta}^{\prime} \sigma \Gamma_{\nu \alpha}^{\prime \lambda} \\
& +g_{\sigma \lambda} \Gamma_{\mu \nu}^{\prime \sigma} \Gamma_{\alpha \beta}^{\prime \lambda},
\end{aligned}
$$

is the fourth rank curvature tensor.

The contravariant curvature tensor $R^{\mu \nu}$ can be obtained by raising the indices ([38], p. 156)

$$
R^{\mu \nu}=g^{\mu \sigma} g^{\nu \lambda} R_{\sigma \lambda}
$$

Following similar methods of V. Fock ([38], p. 425), we have

$$
R^{\mu \nu}=\frac{1}{2} g_{\alpha \beta} \frac{\partial^{2} g^{\mu \nu}}{\partial x^{\prime \alpha} \partial x^{\prime \beta}}-\Gamma^{\prime \mu \nu}+\Gamma^{\prime \mu, \alpha \beta} \Gamma_{\alpha \beta}^{\prime \nu} .
$$

The definition of the invariant of the curvature tensor is $([38]$, p. 425$)$

$$
R \equiv g_{\mu \nu} R^{\mu \nu}
$$

Following similar methods of V. Fock ([38], p. 428), we have

$$
R=g^{\alpha \beta} y_{\alpha \beta}^{\prime}-\Gamma^{\prime \alpha} y_{\alpha}^{\prime}-\Gamma^{\prime}-L^{\prime}
$$

where

$$
\begin{gathered}
y_{\alpha \beta}^{\prime} \equiv \frac{\partial^{2} \lg \sqrt{-g_{0}}}{\partial x^{\prime \alpha} \partial x^{\prime \beta}}, \\
\Gamma^{\prime} \equiv g_{\mu \nu} \Gamma^{\prime \mu \nu},
\end{gathered}
$$




$$
L^{\prime} \equiv-\frac{1}{2} \Gamma_{\alpha \beta}^{\prime \nu} \frac{\partial g^{\alpha \beta}}{\partial x^{\prime \nu}}-\Gamma^{\prime \alpha} \frac{\partial\left(\lg \sqrt{-g_{0}}\right)}{\partial x^{\prime \alpha}}
$$

The second derivative of $\tilde{g}^{\mu \nu}$ is ([38], p. 428)

$$
\begin{aligned}
\frac{\partial^{2} \tilde{g}^{\mu \nu}}{\partial x^{\prime} \alpha x^{\prime \beta}}= & \sqrt{-g_{0}}\left(\frac{\partial^{2} g^{\mu \nu}}{\partial x^{\prime \alpha} \partial x^{\prime \beta}}+y_{\beta}^{\prime} \frac{\partial g_{\mu \nu}}{\partial x^{\prime \alpha}}+y_{\alpha}^{\prime} \frac{\partial g_{\mu \nu}}{\partial x^{\prime \beta}}\right. \\
& \left.+y_{\alpha \beta}^{\prime} g^{\mu \nu}+y_{\alpha}^{\prime} y_{\beta}^{\prime} g^{\mu \nu}\right) .
\end{aligned}
$$

Multiplying $g^{\alpha \beta}$, Eqs. (130) can be written as ([38], p. 428)

$$
\begin{aligned}
g^{\alpha \beta} \frac{\partial^{2} \tilde{g}^{\mu \nu}}{\partial x^{\prime \alpha} \partial x^{\prime \beta}}= & \sqrt{-g_{0}}\left(\frac{\partial^{2} g^{\mu \nu}}{\partial x^{\prime \alpha} \partial x^{\prime \beta}}+2 y^{\alpha} \frac{\partial g^{\mu \nu}}{\partial x^{\prime \alpha}}\right. \\
& \left.+g^{\mu \nu} g^{\alpha \beta} y_{\alpha \beta}^{\prime}+g^{\mu \nu} y_{\alpha}^{\prime} y^{\prime \alpha}\right)
\end{aligned}
$$

Using Eqs. (124) and Eqs. (126), we have ([38], p. 428)

$$
\begin{aligned}
R_{\mu \nu}-\frac{1}{2} g_{\mu \nu} R= & \frac{1}{2}\left(g^{\alpha \beta} \frac{\partial^{2} g_{\mu \nu}}{\partial x^{\prime \alpha} \partial x^{\prime \beta}}+g^{\mu \nu} g^{\alpha \beta} y_{\alpha \beta}^{\prime}\right) \\
& +\frac{1}{2} g^{\mu \nu}\left(\Gamma^{\prime \alpha} y_{\alpha}^{\prime}+\Gamma^{\prime}+L^{\prime}\right) \\
& -\Gamma^{\prime \mu \nu}+\Gamma^{\prime \mu, \alpha \beta} \Gamma_{\alpha \beta}^{\prime} \nu
\end{aligned}
$$

Comparing Eqs. (132) and Eqs. (131), we have ([38], p. 429)

$$
\begin{aligned}
R_{\mu \nu}-\frac{1}{2} g_{\mu \nu} R= & \frac{1}{2 \sqrt{-g_{0}}} g^{\alpha \beta} \frac{\partial^{2} \tilde{g}_{\mu \nu}}{\partial x^{\prime \alpha} \partial x^{\prime \beta}} \\
& +\frac{1}{2} g^{\mu \nu}\left(y_{\alpha}^{\prime} y^{\prime \alpha}+\Gamma^{\prime \alpha} y_{\alpha}^{\prime}+\Gamma^{\prime}+L^{\prime}\right) \\
& -\Gamma^{\prime \mu \nu}+y^{\prime \alpha} \frac{\partial g^{\mu \nu}}{\partial x^{\prime \alpha}}+\Gamma^{\prime \mu, \alpha \beta} \Gamma_{\alpha \beta}^{\prime} \nu .(133)
\end{aligned}
$$

Using the notations defined in Eqs. (79), Eqs. (82), Eqs. (83) and Eqs. (89), Eqs. (133) can also be written as Eqs. (81) ([38], p. 429-430).
[1] C. Moller, The theory of relativity, second edition (Clarendon Press, Oxford, 1972).

[2] S. Weinberg, Gravitation and cosmology (John Wiley \& sons, New York, 1972).

[3] C. Misner, K. Thorne, and J. Wheeler, Gravitation (Freeman, New York, 1973).

[4] R. P. Feynman, Feynman lectures on gravitation, foreword by John Preskill and Kip S. Thorne (AddisonWesley Publishing Company, New York, 1995).

[5] J. Maddox, What remains to be discovered (The Free Press, New York, 1998).

[6] D. Marsh, Phys. Rev. Lett. 118, 011302 (2017).

[7] A. Cho, Science 355, 560 (2017).

[8] A. Einstein, Ann. Math. 40, 922 (1939).

[9] EHTC, The Astrophysical Journal Letters 875, 1 (2019).

[10] P. Hamilton, M. Jaffe, P. Haslinger, Q. Simmons, H. Muller, and J. Khoury, Science 349, 849 (2015).

[11] D. Spergel, Science 347, 1100 (2015).

[12] J. Kim, P. Sikivie, and S. Weinberg, Nature 541, 464 (2017).

[13] A. Bosma, Nature 543, 179 (2017).

[14] E. Gibney, Nature 551, 153 (2017).

[15] E. Whittaker, A History of the Theories of Aether and Electricity, Revised and enlarged edtion, vol. 1 (Thomas Nelson and Sons Ltd., London, 1951).

[16] T. Hirosige, History of physics, in Chinese, translated from the Japanese edtion published in 1968 (Qiushi Press, Beijing, 1968).

[17] L. Kostro, Einstein and the Ether (Apeiron, Montreal, 2000).

[18] P. A. M. Dirac, Direction in Physics (Wiley, New York, 1978).

[19] B. P. Abbott and et al., Phys. Rev. Lett. 116, 061102
(2016).

[20] J. R. Sambles, Science 347, 828 (2015).

[21] N. Cornish, D. Blas, and G. Nardini, Phys. Rev. Lett. 119, 161102 (2017).

[22] D. Giovannini, J. Romero, V. Potocek, G. Ferenczi, F. Speirits, S. Barnett, D. Faccio, and M. Padgett, Science 347, 857 (2015).

[23] A. Einstein, Letters to Solovine 1906-1955 (Citadel, 2000).

[24] M. J. Dupre and F. J. Tipler, International Journal of Modern Physics 21, 1250011 (2012).

[25] X.-S. Wang, Reports on Mathematical Physics 84, 35 (2019).

[26] X.-S. Wang, Progress in Physics 2, 111 (2008).

[27] X.-S. Wang, Progress in Physics 4, 25 (2008).

[28] X.-S. Wang, Physics Essays 27, 398 (2014).

[29] G. Pinch, Handbook of Egyptian Mythology (ABC-CLIO, Inc., Santa Barbara, California, 2002).

[30] B. Riemann, Collected Papers, Translated from the 1892 edition by Roger Baker, Charles Christenson and Henry Orde (Kendrick Press, Heber, 2004).

[31] H. Poincare, The science and hypothesis, translated from the French edtion, in Chinese (Shangwu Press, Beijing, 1997).

[32] J. Taylor, Hidden unity in Nature's laws. Appendix A (Cambridge University Press, 2001).

[33] I. Newton, Mathematical Principles of Natural Philosophy and His System of the World (Univ. of Calif. Press, Berkeley, 1962).

[34] E. Whittaker, A History of the Theories of Aether and Electricity, The Modern Theories 1900-1926 (Thomas Nelson and Sons Ltd., London, 1953).

[35] L. Kelvin, Philosophical Magazine Series 6, 2, 161 (1901). 
[36] H. C. Ohanian and R. Ruffini, Gravitation and Spacetime, 3rd edition (Cambridge University Press, New York, 2013).

[37] L. Liu, The theory of general relativity, in Chinese, 2nd edition (Advanced Education Press, Beijing, 2004).

[38] V. Fock, The Theory of Space Time and Gravitation, 2nd Revised Edition, Translated from the Russian by N. Kemmer (Pergamon Press, London, 1964).

[39] W. Thirring, Annals of Physics 16, 96 (1961).

[40] V. Faraoni, Special relativity (Springer, New York, 2013).
[41] S. V. Babak and L. P. Grishchuk, Physical Review D 61, 024038 (1999).

[42] A. C. Eringen, The Elements of Continuum Mechanics (Robert E. Krieger Pub. Co., Huntington, 1980).

[43] C. M. Will, Theory and Experiment in Gravitational Physics, Revised Edition (Cambridge University Press, Cambridge, 1993).

[44] C. W. Will, Living Rev. Relativity 17, 4 (2014). 